\title{
Diacronie
}

Studi di Storia Contemporanea

$\mathrm{N}^{\circ} 19,3 \mid 2014$

Miscellaneo

\section{Margherita Becchetti, Fuochi oltre il ponte. Rivolte e conflitti sociali a Parma}

Jacopo Bassi

\section{Q OpenEdition \\ Journals}

Edizione digitale

URL: http://journals.openedition.org/diacronie/1659

DOI: $10.4000 /$ diacronie.1659

ISSN: 2038-0925

Editore

Association culturelle Diacronie

Notizia bibliografica digitale

Jacopo Bassi, « Margherita Becchetti, Fuochi oltre il ponte. Rivolte e conflitti sociali a Parma », Diacronie

[Online], $N^{\circ}$ 19, 3 | 2014, documento 12, Messo online il 01 septembre 2014, consultato il 24

septembre 2020. URL : http://journals.openedition.org/diacronie/1659; DOI : https://doi.org/10.4000/ diacronie. 1659 


\section{RECENSIONE:}

\section{Margherita BECCHETTI, Fuochi oltre il ponte. Rivolte e conflitti sociali a Parma, Roma, DeriveApprodi, 2013, 303 pp.}

a cura di Jacopo BASSI *

Il libro di Margherita Becchetti, dottore di ricerca in Storia presso l'Università di Parma e membro del Centro Studi Movimenti Parma, è incentrato sulla vita sociale nell'Oltretorrente, la cui fama ribellistica ha dato vita ad una vasta letteratura, che ha conosciuto un'ulteriore fioritura anche in anni recenti ${ }^{1}$. Il nome dell'Oltretorrente è inevitabilmente legato con le barricate antifasciste di Parma, climax e ultimo atto dell'antifascismo negli anni Venti, sorte sulla scorta dello sciopero legalitario organizzato in risposta alla marcia su Roma. L'epopea del ribellismo dei quartieri popolari di Parma, che fu in grado di respingere le diecimila camicie nere guidate da Italo Balbo, ha generato un gran numero di letture storiografiche ${ }^{2}$. Il lavoro di Becchetti, tuttavia, ha precisi caratteri di originalità dal momento che si sofferma sul periodo precedente a quello che ha reso noti i borghi dell'Oltretorrente: l'attenzione è infatti rivolta alla formazione dei caratteri peculiari del ribellismo dei borghi, dagli anni immediatamente successivi all'unificazione italiana all'entrata in guerra dell'Italia.

Il libro colma certamente un vuoto storiografico, contribuendo a gettare un ponte tra il periodo pre-unitario e quello degli anni Venti, già preso in esame dalla storiografia.

\footnotetext{
${ }^{1}$ Ad esempio GIUFFREDI, Massimo (a cura di), Nella rete del regime : gli antifascisti del Parmense nelle carte di polizia, 1922-1943, Roma, Carocci, 2004 e il libro - documentario di Giancarlo Bocchi: BOCCHI, Giancarlo, Il ribelle : Guido Picelli, una vita da rivoluzionario, Pomezia, STR Press, 2013, oltre al romanzo Oltretorrente di Pino Cacucci (CACUCCI, Pino, Oltretorrente, Milano, Feltrinelli, 2003).

${ }^{2}$ Sull'argomento, si veda: GAMBETTA, William, GIUFFREDI, Massimo (a cura di), Memorie d'agosto. Letture e immagini delle Barricate antifasciste di Parma del 1922, Milano, Punto rosso, 2007.
} 
Una delle maggiori difficoltà incontrate dalla autrice, come sottolinea Antonio Parisella nella sua prefazione, è stato quello di non trovare fonti documentarie strutturate e omogenee. Tuttavia, da questa apparente difficoltà ha sicuramente tratto giovamento l'originalità della ricerca (non appiattita su piste già battute) e la qualità della narrazione. L'esposizione di Margherita Becchetti alterna infatti i documenti archivistici alle cronache dei giornali; questa combinazione sortisce l'effetto di restituire ai lettori una percezione molteplice degli eventi.

L'autrice chiarisce sin dall'introduzione i suoi intenti:

\begin{abstract}
Non ho voluto fare una storia del movimento operaio, né una storia delle sue organizzazioni, quanto mostrare la relazione continua, difficile e certamente instabile tra la turbolenza delle classi popolari e le culture politiche che, per lo meno dalla seconda metà dell'Ottocento, hanno cercato di contendersi un ruolo di guida sulla via della loro emancipazione3.
\end{abstract}

Emerge quindi nelle pagine un affresco punteggiato dalle miniature dei popolani dei borghi; ed è «anche attraverso i suoi atti di insubordinazione e di sfida ai potenti di turno, [che] quel popolo è diventato comunità»4. Non si tratta, però, di una storia della marginalità, quanto, piuttosto, di una storia della conflittualità prodotta dalla marginalità. La struttura del libro si divide si snoda intorno a tre capitoli principali.

Il primo di questi è consacrato ad una ricostruzione delle vicende storiche alla base della costituzione dell'Oltretorrente e alle condizioni economiche e sociali venutesi a creare in forza dell'evoluzione storica; in queste pagine la descrizione storica si sovrappone a quella geografica - o, sarebbe meglio dire, urbanistica - restituendo al lettore un'immagine precisa dei borghi.

Nel secondo capitolo, incentrato sul tema delle rivolte nel trentennio 1868-1898, attraverso le fonti archivistiche e i giornali dell'epoca vengono ricostruiti i disordini durante gli anni dei governi Crispi, Rudinì e Pelloux, contraddistinti da una polica reazionaria e fortemente repressiva.

Proprio in questo ambiente cominciarono a farsi strada nuove idee che modificarono i caratteri del conflitto sociale. Nacquero forme di organizzazione politica, inizialmente tra il ceto artigianale, grazie alle società di mutuo soccorso:

3 BECCHETTI, Margherita, Fuochi oltre il ponte. Rivolte e conflitti sociali a Parma (1868-1915), Roma, DeriveApprodi, 2013, p. 17.

4 Ibidem, p. 22. 
[...] in città cominciarono a distinguersi nuovi luoghi, piccole stanze di case private trasformate in spazi comuni in cui poter discutere, in cui riunirsi quando il prefetto vietava gli assembramenti o i cortei, in cui ascoltare uomini appassionati parlare di unione operaia e dominio borghese. [...] Numerosi popolani cominciarono a frequentare queste stanze, che divennero così il teatro di una vigorosa educazione politica e, al tempo stesso, disseminarono altre abitudini e modalità di gestione del conflitto5.

Pur mantenendo una certa distanza fra loro (alla lunga le organizzazioni socialiste, si dimostrarono meno concilianti rispetto a quelle anarchiche riguardo all'indisciplina delle popolazione dei borghi) le strutture e l'attivismo politico svolsero un ruolo fondamentale nell'evoluzione del ribellismo dell'Oltretorrente:

Furono dunque queste organizzazioni, sebbene con i loro limiti, a rappresentare la prima scuola di lotta politica per le classi popolari della città e, sul loro ordito, i primi attivisti socialisti e anarchici iniziarono a tessere la loro propaganda ${ }^{6}$.

Il capitolo successivo, incentrato sul periodo 1898-1915, è segnato dagli scioperi (nazionali e locali), dalla mobilitazione politica e sindacale e dalla conseguente aumentata politicizzazione del ribellismo dell'Oltretorrente. Alle vicende di respiro nazionale (la settimana rossa, le «radiose giornate») che vengono analizzate nel loro respiro locale, si alternano le vicende parmigiane (come lo sciopero agrario del 1908).

Durante questi anni:

[...] il ribellismo urbano trovò nell'azione diretta una pratica di mobilitazione a sé conforme e intorno alla Camera del Lavoro si raccolse l'intero popolo dei borghi che - se anche non figurava iscritto negli elenchi dei suoi organizzati - assicurava ai suoi dirigenti quella forza d'urto che rese possibili anche le iniziative più ambiziose e più stupefacenti, come il grande sciopero agrario del 1908 o le imponenti manifestazioni contro la guerra di Libia nel 19127.

L'ultima sezione del libro tenta, per ammissione della stessa Becchetti di ridisegnare il volte dei rivoltosi parmensi; discostandosi dalle altre pagine, la narrazione cede il passo ad un'analisi maggiormente attenta al dato quantitativo.

5 Ibidem, p. 146.

${ }^{6}$ Ibidem, p. 148.

7 Ibidem, p. 199. 
Chiude il libro un epilogo in cui vengono individuate le continuità (la ripetizione di una sorta di repertoire nelle rivolte a Parma, quasi a ripetizione di un canovaccio ben conosciuto) e le discontinuità (individuabili nelle modalità e negli obiettivi dei cortei che partivano dall'Oltretorrente) nella messa in scena della protesta a Parma durante questo periodo.

Ciò che emerge con maggior forza nella disamina dei fatti - e costituisce un filo conduttore della ricerca - è:

[...] un elemento di fondo costante nel tempo, [...] l'insofferenza delle classi popolari verso l'autorità, la loro avversione verso le forze dell'ordine, una certa voglia di «menar le mani», di misurarsi con la «sbirraglia», di far orgogliosa mostra della propria capacità di conflitto, di vivere e praticare il proprio mito ${ }^{8}$.

Uno dei pregi maggiori del libro è la sapiente miscela con cui l'autrice alterna una precisa disamina della documentazione con una narrazione storica che riesce nell'impresa di restituire le condizioni di vita e sociali dei borghi dell'Oltretorrente, andando alla radice delle ragioni delle rivolte. Tutto ciò senza ricadere in schematismi $o$ forzature interpretative ma, semplicemente facendo "parlare i documenti". Se il libro riesce così nell'intento di ricostruire le premesse all'epopea delle barricate a Parma ha il merito - ancora maggiore - di costituire una narrazione storica a sé stante, indipendente dal peso storico (e storiografico) degli eventi del 1922, in grado, per molti anni, di catalizzare gli studi e le attenzioni di politici e storici, adombrando la ricerca sul periodo precedente.

\footnotetext{
8 Ibidem, p. 19.
} 


\section{* L'autore}

Jacopo Bassi è Dottore magistrale in Storia contemporanea (Università degli Studi di Bologna). Nel corso dei suoi studi si è occupato di storia della Grecia e dell'Albania in età contemporanea e di storia e istituzioni della Chiesa ortodossa. Ha lavorato per le case editrici Éditions des femmes e Il Mulino; attualmente è redattore presso la casa editrice Zanichelli.

\section{Per citare questo articolo:}

BASSI, Jacopo, «Recensione: Margherita BECCHETTI, Fuochi oltre il ponte. Rivolte e conflitti sociali a Parma, Roma, DeriveApprodi, 2013, 303 pp.», Diacronie. Studi di Storia Contemporanea, 29/09/2014,

URL:< http://www.studistorici.com/2014/09/29/bassi_numero_19/ >

Diacronie Studi di Storia Contemporanea 3 www.diacronie.it

Risorsa digitale indipendente a carattere storiografico. Uscita trimestrale.

redazione.diacronie@hotmail.it

Comitato di redazione: Jacopo Bassi - Luca Bufarale - Elisa Grandi - Deborah Paci - Fausto Pietrancosta - Matteo Tomasoni - Luca Zuccolo 